\title{
Anomalias de calendário no mercado brasileiro: uma análise com empresas pertencentes ao IGC
}

\section{Luciano Ferreira Carvalho}

Doutorando em Economia pelo Instituto de Economia

da Universidade Federal de Uberlândia

lucianofc@netsite.com.br

\section{Rodrigo Fernandes Malaquias}

Doutorado em Administração de Empresas pela

Fundação Getulio Vargas (FGV)

Professor efetivo da Universidade Federal de Uberlândia

rodrigofmalaquias@yahoo.com.br
Contextus

ISSNe 2178-9258

ISSN 1678-2089

Organização: Comitê Científico Interinstituciona Editor Científico: José Carlos Lázaro Avaliação: Double Blind Review pelo SEER/OJS Revisão: Gramatical, normativa e de formatação Recebido em 18-02-2011 Aprovação Final em 15-12-2012

\section{RESUMO:}

O objetivo principal deste trabalho foi analisar o efeito calendário no mercado de ações brasileiro. Para tanto, foram selecionas as companhias pertencentes ao Índice de Governança Corporativa (IGC), no período de janeiro/2003 a dezembro/2007. Com dados de 41 empresas, selecionadas com base no indicador de liquidez, os principais resultados mostraram-se alinhados com a teoria sobre o assunto, a Hipótese de Mercado Eficiente. Em outras palavras, não houve indícios de padrões temporais para a maior parte das observações analisadas, seja em relação ao efeito dia-da-semana ou ao efeito janeiro.

Palavras-chave: Efeito dia-da-semana. Efeito janeiro. Hipótese de Mercado Eficiente.

\section{ABSTRACT:}

The aim of this research was to analyze the calendar effect in the Brazilian capital market. To do so, we selected the companies pertaining of the corporate governance index, in the period from January/2003 to December/2007. Based on the liquidity indicator, we selected for the study 41 companies, and the main results are aligned with the theory about this issue: the Market Efficiency Hypothesis. In other words, we didn't found evidences of temporal patterns (day-of-week or January effects) for the major part of the observations.

Keywords: Day-of-week effect; January effect; Market Efficiency Hypothesis. 


\section{INTRODUÇÃO}

O estudo sobre o comportamento do preço das ações no mercado de capitais iniciou-se no século XX, tendo Bachelier (1990) como precursor. A origem do estudo esteve relacionada com a análise do passeio aleatório dos preços (random walk), em que as variações nos preços das ações seriam aleatórias. Kendall (1953) também desenvolveu outro trabalho relevante sobre o assunto, em que concluiu que o comportamento dos preços das ações segue um passeio aleatório. Embora tais pesquisas sejam um marco sobre o tema, a teoria que sustenta essa discussão surgiu com os estudos de Eugene F. Fama e Paul A. Samuelson, na década de 1960, conhecida como Hipótese de Eficiência de Mercado (HEM). Conforme destacam Bampi, Colombo e Kloeckner (2011, p. 2), a HEM representa "[...] um dos principais pilares da Teoria Moderna de Finanças".

De acordo com a HEM, as informações necessárias para os investidores tomarem suas decisões estariam refletidas no preço das ações, basicamente, em três formas: fraca, semiforte e forte. Nesse contexto, Fama (1970) destaca três formas de eficiência de mercado: i) forma fraca, em que a negociação de ações com base em informações históricas sobre seus preços ou volume de negociação não agregaria valor; ii) forma semiforte, em que a negociação de ações com base em informações públicas não agregaria valor; iii) forma forte, em que a negociação de ações com base em quaisquer tipos de informações (públicas ou internas das empresas) não agregaria valor. Com isso, testes empíricos são desenvolvidos por diferentes autores e em diferentes mercados de capitais com o objetivo de verificar se, de alguma maneira, há indícios de que a negociação de ações com base em informações (históricas, públicas ou até mesmo privadas) agrega valor aos investidores. A HEM é refutada quando informações podem ter proporcionado retornos anormais. Um padrão regular de retorno de um ativo no mercado de capitais, e que é confiável, amplamente conhecido e inexplicável, é conhecido como anomalia (LO, 2007), pois permite certo grau de previsibilidade, regularidade e confiabilidade, garantindo a investidores vantagem com base em informações, o que contraria a HEM.
Autores como Gibbons e Hess (1981), Keim e Stambaugh (1984), Kato e Schallheim (1985), Harris (1986), Fama e French (1988), Keim (1989), Chan e Chen (1991), Amihud e Mendelson (1991), Chan, Hamao e Lakonishok (1991) já documentaram em seus estudos a ocorrência de anomalias no mercado de capitais, especificamente relacionadas com alguns dias, com o dia da semana ou o mês do ano ou com as características das empresas, tal como o valor de mercado. Em outras palavras, de acordo com os estudos mencionados, a negociação de ações com base em informações sobre, por exemplo, o dia da semana em que poderá ser feita a negociação (ou qual mês) ou sobre quais as características das empresas estariam relacionadas às variações de seus preços, podem agregar valor aos investidores, o que, conforme já apontado, contraria a HEM.

Sob essa ótica, este estudo se encaixa na linha de pesquisas que abordam a HEM, particularmente sobre questões relacionadas a anomalias de calendário (efeito dia-da-semana e efeito mês-do-ano). A partir de 2001, as empresas brasileiras passaram a contar com novas exigências relacionadas às boas práticas de governança corporativa. As empresas que atendem aos requisitos e se enquadram no Índice de Governança Corporativa (IGC) tenderiam a possuir melhores níveis de informações em seus relatórios contábeis como também maior transparência no relacionamento com seus shareholders.

"A valorização e a liquidez das ações são influenciadas positivamente pelo grau de segurança oferecido pelos direitos concedidos aos acionistas e pela qualidade das informações prestadas pelas companhias" (CAMPOS, LAMOUNIER e BRESSAN, 2011, p. 8); assim, a análise de potenciais anomalias de mercado para empresas listadas no IGC pode proporcionar novas discussões sobre retornos decorrentes de informações públicas para empresas predispostas a garantir melhores níveis de qualidade de informações disponibilizadas aos seus usuários.

Diante das novas exigências às quais as empresas brasileiras pertencentes ao IGC estiveram sujeitas, procurou-se, neste estudo, responder à seguinte questão de pesquisa: há indícios de padrões temporais de retorno 
no mercado de capitais brasileiro, considerando-se as empresas componentes do IGC? Dessa forma, o objetivo geral deste estudo é analisar a existência de padrões temporais de retorno no mercado de capitais brasileiro, levando-se em conta, especificamente, as empresas componentes do IGC. O período considerado para análise foi entre 02/01/2003 e 31/12/2007, tendo como base os dados disponíveis no Banco de Dados ECONOMÁTICA relativos às empresas listadas em qualquer um dos níveis de governança. A escolha do período após 2003 deveuse ao número de ações disponíveis; aumentar o período implicaria na redução da amostra, por isso optou-se por uma janela de cinco anos. Destaca-se também que nem todas as empresas pertencentes ao IGC foram contempladas no estudo em decorrência do fator liquidez (ou seja, falta de negociação diária de algumas ações).

A hipótese nula implícita no objetivo do trabalho é a seguinte: H0 - não há padrões temporais de retorno. Assim, padrões de retorno tais como efeito janeiro e efeito dia-da-semana não devem existir para as empresas e o período analisados, o que está em linha com a HEM. Como esse segmento de mercado ainda não foi exclusivamente examinado em pesquisas sobre o assunto, as evidências apontadas por esta pesquisa podem contribuir para o debate da teoria ligada à HEM. Camargos e Barbosa (2003) também já ressaltaram que ainda são necessários estudos sobre a eficiência do mercado brasileiro, especialmente após 1994, ano que representa um marco para uma relativa estabilidade econômica e monetária no Brasil.

Além desta introdução, este estudo divide-se em mais quatro seções. Na próxima seção, apresenta-se a revisão da bibliografia, abrangendo padrões temporais de retorno, anomalias de valor e evidências empíricas no mercado brasileiro. Na terceira seção são detalhados os aspectos metodológicos relacionados ao estudo, e, na quarta, mostra-se a análise dos resultados. A quinta e última seção contém as considerações finais e recomendações para pesquisas futuras

\section{ANOMALIAS DE CALENDÁRIO}

Segundo Lo (2007), talvez o mais comum desafio ao mercado eficiente sejam as anomalias, padrões regulares em um retorno de ativo, os quais são confiáveis, amplamente conhecidos e inexplicáveis. A regularidade e a confiabilidade desses padrões refletem um grau de previsibilidade, enquanto o fato de eles serem amplamente conhecidos implica que investidores podem levar vantagem disso. Conforme já abordado, tem-se a rejeição da HEM quando informações históricas, públicas e, até mesmo, internas podem proporcionar retornos anormais aos investidores.

Entre as anomalias existentes, podem ser destacadas duas: i) as anomalias de calendário, nas quais os retornos das ações mostram padrões temporais, como retornos mais baixos ou mais altos em certos dias, semanas ou meses do ano; ii) as anomalias que relacionam retornos a características de empresas, como o tamanho, o quociente entre valor de mercado e valor patrimonial, entre outras. Este estudo aborda, especificamente, as anomalias de calendário, das quais se ressaltam dois padrões: o efeito dia-da-semana e o efeito janeiro.

Conforme o próprio nome sugere, o efeito dia-da-semana se relaciona às diferenças significativas entre retornos de diferentes dias da semana. Segundo Gibbons e Hess (1981, p. 579),

Pesquisadores geralmente assumem que a distribuição de retornos de ações é idêntica para todos os dias da semana... Entretanto, há razões para suspeitar que a distribuição de retornos possa variar de acordo com o dia da semana; o mais obvio é o impacto do fim de semana no retorno da segunda-feira.

Os resultados de Gibbons e Hess (1981) e de Keim e Stambaugh (1984), tal como os obtidos por Harris (1986), apontam que os retornos das segundas-feiras são significativamente diferentes e menores em comparação àqueles obtidos nos demais dias da semana, o que pode ser potencialmente explicado pelo reflexo que a segunda-feira traz do fim de semana. Por outro lado, os retornos no mês de janeiro são, em média, maiores que os retornos dos outros meses do ano (FAMA, 1991; ELTON et al., 2004). Fama (1991) e Elton et al. (2004) também documentaram uma relação significativa entre o porte das empresas e o efeito janeiro, em que as ações de 
empresas menores, no mês de janeiro, tendem a registrar melhor rentabilidade. Embora já se tenham registrado tais anomalias no mercado, Kato e Schalleim (1985), com dados do mercado de ações japonês, concluíram que as anomalias de calendário e de características da empresa são sensíveis ao tipo de índice utilizado nas análises.

Questões fiscais podem ter papel importante na explicação das anomalias de mercado. Por exemplo, o efeito janeiro pode ser influenciado pela decisão dos investidores de, nos últimos dias do ano, vender títulos que apresentaram perdas; no início do ano, essa venda pode ser compensada pela aquisição de títulos equivalentes. A posição do investidor sofre pequena variação, mas, fiscalmente, no ano que se encerrou, ele apresentou uma perda

A anomalia dia-da-semana representa um dos efeitos mais estudados no mercado brasileiro, já encontrado por autores como: Lemgruber, Becker e Chaves (1988), Costa Jr. (1990), Costa Jr. e Lemgruber (1993), Bone e Ribeiro (2002) e Pagnani e Olivieri (2004). Por outro lado, resultados obtidos por autores como Costa Jr. e Ceretta (2000) e Leal e Sandoval (1994) não apontaram indícios relativos a tal anomalia.

Em relação ao efeito janeiro, em estudos como os de Bonomo e Agnol (2002) e Pagnani e Olivieri (2004), foram apontadas evidências desse efeito; já os estudos de Costa Jr. (1990), Costa Jr. e O’Hanlon (1991), Leal e Sandoval (1994) e Santos et al. (2007) não registraram retornos anormais significativos no mês de janeiro. Para resumir os resultados de alguns dos estudos empíricos já desenvolvidos no Brasil sobre as anomalias de calendário, foi elaborado o Quadro 1, a seguir.

Quadro 1 - Estudos empíricos no Brasil sobre anomalias temporais e de valor

\begin{tabular}{|c|c|l|}
\hline Anomalias & Resultados & \multicolumn{1}{c|}{ Autores } \\
\hline \multirow{2}{*}{ Dia-da-semana } & $\begin{array}{c}\text { Encontram } \\
\text { esse padrão }\end{array}$ & $\begin{array}{l}\text { Lemgruber, Becker e Chaves (1988); } \\
\text { Costa Jr. (1990); Costa Jr. e } \\
\text { Lemgruber (1993); Bone e Ribeiro (2002); } \\
\text { Pagnani e Olivieri (2004). }\end{array}$ \\
\cline { 2 - 4 } & $\begin{array}{c}\text { Não encontram } \\
\text { esse padrão }\end{array}$ & $\begin{array}{l}\text { Leal e Sandoval (1994); } \\
\text { Costa Jr e Ceretta (2000) }\end{array}$ \\
\hline \multirow{2}{*}{$\begin{array}{c}\text { Mês do ano } \\
\text { (efeito Janeiro) }\end{array}$} & $\begin{array}{c}\text { Encontram } \\
\text { esse padrão }\end{array}$ & $\begin{array}{l}\text { Bonomo e Agnol (2002); } \\
\text { Pagnani e Olivieri (2004). }\end{array}$ \\
\cline { 2 - 4 } & $\begin{array}{c}\text { Não encontram } \\
\text { esse padrão }\end{array}$ & $\begin{array}{l}\text { Costa Jr. (1990); Leal e Sandoval (1994); } \\
\text { Costa Jr. e O’Hanlon (1991); } \\
\text { Santos et al. (2007). }\end{array}$ \\
\hline
\end{tabular}

Costa Jr. e Caretta (2000), apesar de não terem detectado padrões temporais no mercado brasileiro, perceberam evidências de tais efeitos em mercados de outros países, como México, Peru e Venezuela. Vale destacar também o trabalho de Fajardo e Pereira (2008), em que foram analisadas anomalias de calendário nos retornos do Ibovespa. Os autores concluíram que, para o período como um todo (1995 a 2007), há indícios de ocorrência das anomalias. Contudo, para períodos mais recentes (após 1999), os indícios da presença do efeito calendário são não significativos, o que pode auxiliar na compreensão de resultados divergentes entre diferentes pesquisas. Os autores sugerem como potencial explicação para esses resultados o fato de que "[...] o Brasil passou a ter estabilidade há pouco tempo atrás", situação diferente da de outros mercados de capitais, como o mercado norte-americano (FAJARDO e PEREIRA, 2008, p. 252). Assim, com investidores mais atentos às informações e oportunidades de negociação no Brasil, o equilíbrio tende a ficar mais estável ao longo do tempo, dificultando a obtenção de retornos anormais com base em dados públicos.

\section{ASPECTOS METODOLÓGICOS}

\subsection{Amostra}

Conforme já destacado na introdução deste estudo, foram analisados os dados referentes às empresas que estão no IGC, e as cotações, acessadas por meio do Banco de Dados ECONOMÁTICA. O período de análise envolve dados diários de cinco anos (02/01/2003 a 31/12/2007), escolhido por representar uma época de valorização da bolsa de valores, correspondente a $484 \%$ para o Índice BOVESPA. A inclusão de períodos anteriores implicaria na redução do número de empresas da amostra.

$\mathrm{Na}$ escolha das empresas investigadas, levou-se em consideração o critério "liquidez", pois a variação do preço de uma ação está diretamente relacionada com o nível de negociação desse título na Bolsa de Valores (LIMA e TERRA, 2004). A representação matemática do indicador de liquidez utilizado está disponível na Equação 1, a seguir.

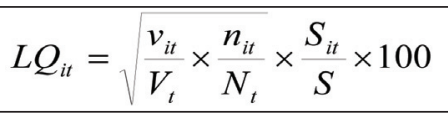


Em que: Indicador de liquidez da ação i para o período t; Número de pregões no período t, em que se constatou pelo menos 1 negócio com ação i no mercado à vista; Número de negócios com a ação i verificados no mercado à vista da BOVESPA no período t; Número de negócios registrados no mercado à vista da BOVESPA no período $\mathrm{t} ; \mathrm{V}_{t t}=$ Volume financeiro gerado pelos negócios com a ação i no mercado à vista no período $\mathrm{t} ; \mathrm{V}_{t}=$ Volume financeiro total registrado no mercado à vista da BOVESPA para o período t.O corte utilizado para o indicador de liquidez foi o valor de 0,015. Com base na lista de 179 empresas que fazem parte do IGC, foram eliminadas 138 por terem sido incluídas no índice após o ano de 2003 ou por não satisfazerem ao critério de liquidez. Dessa forma, fizeram parte da amostra final para o estudo 41 empresas, cuja identificação está disponível no Apêndice A deste trabalho. A Tabela 1 apresenta algumas informações descritivas relativas às empresas $\mathrm{e}$ ao período selecionado.

Tabela 1 - Informações descritivas sobre as empresas da amostra

\begin{tabular}{|lccccc|}
\hline Variáveis & n & Média & Desv. Padr. & Mínimo & Máximo \\
\hline Ativo Total Cons. Dez;05 (RS mil) & 41 & 29.122 .429 & 58.095 .088 & 674.931 & 252.976 .988 \\
Segmento - Nivel 1 & 30 & - & - & - & - \\
Segmento - Nivel 2 & 05 & - & - & - & - \\
Segmento - Novo Mercado & 06 & - & - & - & - \\
Qtd. Segmentos-Feiras (por empresa) & 228 & - & - & - & - \\
\hline
\end{tabular}

Fonte: Ativo Total Consolidado - Banco de Dados ECONOMÁTICA.

O Gráfico 1 permite que sejam visualizadas as empresas em relação ao setor de atuação ao qual estão vinculadas.

Gráfico 1 - Setores de atuação vinculados às empresas da amostra

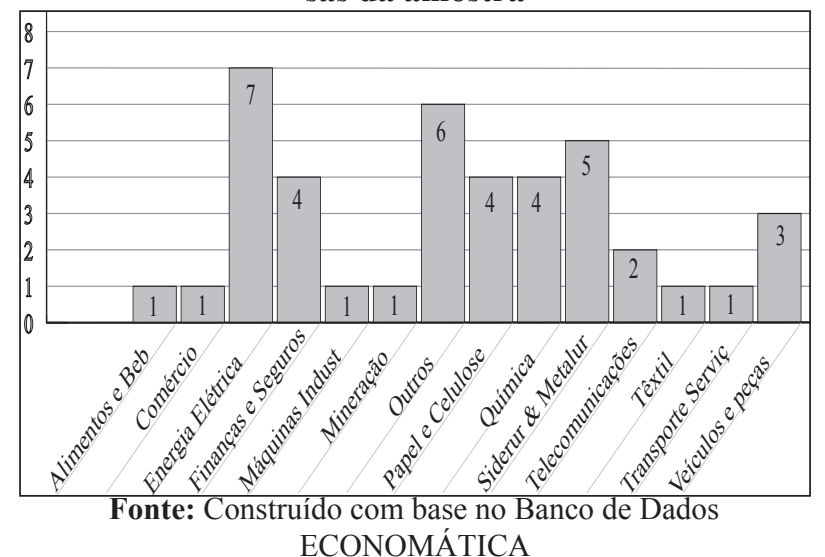

Destaca-se que, entre as empresas escolhidas, foi analisada apenas uma classe de ações de cada instituição (ON, PN, PNA, PNB etc.); o critério utilizado para segregar a classe de ações foi o indicador de liquidez, e as selecionadas foram aquelas com maior índice. Para as análises quantitativas, considerou-se o logaritmo natural dos retornos diários e mensais das ações selecionadas.

\subsection{Modelos econométricos utilizados}

O método utilizado para analisar as anomalias foi a regressão múltipla com variáveis dummy. No que se refere ao efeito dia-da-semana, utilizou-se a Equação 2, a seguir.

$R_{t}=\alpha+\beta_{1} \operatorname{Seg}+\beta_{2}$ Ter $+\beta_{3} Q u i+\beta_{4} \operatorname{Sex}+\beta_{5} R_{m}$

Em que: Rt é o retorno diário das ações da amostra; Rm é o retorno do mercado; Seg., Ter., Qui. e Sex. são variáveis dummy que assumiram valores zero (0) ou um (1) e medem as diferenças de retorno entre os respectivos dias e a quarta-feira $(\alpha)$.

Ainda na Equação 2, tem-se a variável Rm, que se refere ao retorno de mercado. Ela foi adicionada ao modelo com o objetivo de se contornar o potencial problema decorrente de omissão de variável relevante. A Equação 3, abaixo, descreve o modelo utilizado para analisar o efeito janeiro.

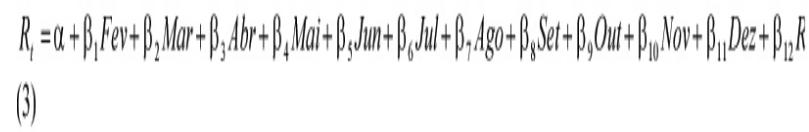

Em que: Rt é o retorno mensal das ações da amostra; Rm é o retorno do mercado; fev., mar., abr., maio, jun., jul., ago., set., out., nov., e dez. são variáveis dummy que assumiram valores zero (0) ou um (1) e medem as diferenças de retorno entre os respectivos meses e o mês de janeiro $(\alpha)$.

Na Equação 3 também foi incluído o retorno de mercado, visando-se novamente evitar o problema de omissão de variável relevante. A próxima seção descreve os resultados obtidos com a aplicação das regressões.

\section{RESULTADOS}

Após a coleta, a tabulação e o cálculo dos logaritmos naturais dos retornos das ações pertencentes à amostra, os parâmetros das regressões multivariadas foram 
estimados, e os resultados estão resumidos nos Apêndices $\mathrm{B}$ e $\mathrm{C}$ deste trabalho, referindo-se, respectivamente, à análise do efeito dia-da-semana e ao efeito janeiro. Ressalta-se a utilização tanto de procedimentos robustos para a correção da heteroscedasticidade (quando necessária), como também dos erros-padrão Newey-West, na presença de autocorrelação, para as estimativas feitas por Mínimos Quadrados Ordinários (WOOLDRIDGE, 2006).

Os resultados obtidos para a amostra e para o período analisados apontam evidências alinhadas à HEM, ou seja, parece não haver indícios significativos de padrões temporais de retorno, especificamente os efeitos diada-semana e janeiro. No Apêndice B, pode-se conferir que, entre diferentes dias da semana, houve diferenças significativas de retorno apenas para seis casos, correspondentes a 5\%. Tais diferenças não mostram um padrão recorrente, o que está de acordo com a HEM.

No Apêndice $\mathrm{C}$, nota-se que não houve presença de padrão entre os betas relativos às variáveis dummies; destaca-se também que, das 41 ações analisadas, apenas dez apresentaram retornos anormais significativos no mês de janeiro, na maioria negativos. Tal como as evidências para o efeito dia-da-semana, a HEM não pode ser rejeitada para essa análise. Testes adicionais foram conduzidos para verificar se o setor das empresas apresentava alguma relação com os betas significativos identificados, mas nenhuma relação significativa foi observada. Em outras palavras, o setor das empresas parece não ter relação com os efeitos dia-da-semana e janeiro.

Mesmo ainda sendo necessárias pesquisas no Brasil sobre a eficiência do mercado de capitais do país (CAMARGOS e BARBOSA, 2003), não há indícios de que a negociação com base em informações públicas possa gerar retornos anormais significativos para os investidores. De uma maneira geral, os resultados estão alinhados com aqueles previamente encontrados por Costa Jr. (1990), Costa Jr. e O’Hanlon (1991), Leal e Sandoval (1994), Costa Jr. e Ceretta (2000) e Santos et al. (2007). Contudo, este trabalho estende a discussão por levar em consideração exclusivamente empresas pertencentes ao IGC, o que pode auxiliar para o debate da teoria que vem sendo construída sobre o assunto no mercado brasileiro.

\section{CONSIDERAÇÕES FINAIS}

Com base na teoria relativa à HEM, analisou-se neste estudo o efeito calendário no mercado brasileiro para empresas pertencentes ao IGC. O efeito calendário proporcionaria aos investidores a possibilidade de obtenção de retornos anormais significativos apenas com base em informações sobre o dia-da-semana para negociar ou o mês em que algum padrão específico se repetiria. Considerando o efeito calendário como uma das anomalias de mercado, na ocorrência dele tem-se evidências contrárias à HEM.

Contudo, mesmo com estudos brasileiros previamente realizados e que encontraram tal efeito (LEMGRUBER, BECKER e CHAVES, 1988; COSTA JR., 1990; COSTA JR. e LEMGRUBER, 1993; BONE e RIBEIRO, 2002; BONOMO e AGNOL, 2002; PAGNANI e OLIVIERI, 2004), neste estudo os resultados obtidos mostraramse alinhados com a HEM; ou seja, o efeito calendário aconteceu em poucos casos, não garantindo que os investidores poderiam obter vantagens com negociações baseadas nesses padrões. Resultados similares já foram obtidos por outros estudos (COSTA JR, 1990; COSTA JR. e O'HANLON, 1991; LEAL e SANDOVAL, 1994; COSTA JR. e CERETTA, 2000; e SANTOS et al., 2007), mas não especificamente para companhias pertencentes ao IGC.

Em um sentido econômico, os resultados obtidos podem ser interpretados da seguinte forma: quando investidores negociam ações com base apenas em informações relativas ao dia da semana ou ao mês do ano, eles podem até obter retorno anormal. Contudo, em média, os retornos anormais positivos não são significativamente diferentes de zero, indicando que essa estratégia nem sempre garantirá aos investidores retornos positivos e significativos.

Uma vez que aqui foram levadas em consideração apenas as anomalias de calendário, sugere-se, para pesquisas futuras, a análise em amostra equivalente à utilizada nesta pesquisa - $\mathrm{a}$ das anomalias relacionadas às características 
das empresas. Sugere-se também ponderar diferentes períodos de corte na amostra, incluindo na análise diferentes cenários econômicos aos quais os investidores e o próprio mercado estiveram sujeitos. Por exemplo, para o período, houve várias ocorrências em que o retorno acumulado do Ibovespa não foi crescente.

\begin{tabular}{|c|c|c|c|c|}
\hline \multicolumn{5}{|c|}{ Apêndice A - Empresas/Ações Componentes da Amostra } \\
\hline Cod. & Nome Completo & Segmento & Setor & $\begin{array}{c}\text { Ativo Total Cons. } \\
\text { Dez./05 (R\$ mil) }\end{array}$ \\
\hline ALPA4 & Sao Paulo Alpargatas S.A. & $\mathrm{N} 1$ & Textil & 1.138 .358 \\
\hline ARCZ6 & Aracruz Celulose S.A. & $\mathrm{N} 1$ & Papel e Celulose & 9.378 .735 \\
\hline BBDC4 & Banco Bradesco S.A. & N1 & Finanças e Seguros & 208.682 .930 \\
\hline BRAP4 & Bradespar S.A. & $\mathrm{N} 1$ & Outros & 6.347 .325 \\
\hline BBAS3 & Banco do Brasil S.A. & NM & Finanças e Seguros & 252.976 .988 \\
\hline BRTP4 & Brasil Telecom Participacoes S.A. & N1 & Telecomunicações & 18.202 .788 \\
\hline BRTO4 & Brasil Telecom S.A. & $\mathrm{N} 1$ & Telecomunicações & 16.728 .089 \\
\hline BRKM5 & Braskem S.A. & N1 & Química & 15.590 .751 \\
\hline CCRO3 & Companhia de Concessoes Rodoviarias & NM & Transporte Serviç & 3.424 .538 \\
\hline CLSC6 & Centrais Eletricas de Santa Catarina S.A. & $\mathrm{N} 2$ & Energia Elétrica & 3.358 .753 \\
\hline CMIG4 & Cia Energ Minas Gerais - CEMIG & N1 & Energia Elétrica & 19.839 .585 \\
\hline CESP5 & Cesp - Companhia Energetica de Sao Paulo & $\mathrm{N} 1$ & Energia Elétrica & 19.474.374 \\
\hline CNFB4 & Confab Industrial S.A. & $\mathrm{N} 1$ & Siderur \& Metalur & 1.126 .533 \\
\hline DURA4 & Duratex S.A. & N1 & Outros & 829.878 \\
\hline ELET6 & Centrais Elet Brasileiras S.A. & N1 & Energia Elétrica & 1.538 .592 \\
\hline ELPL5 & Eletropaulo Metropolitana El.S.Paulo S.A. & $\mathrm{N} 2$ & Energia Elétrica & 12.377 .318 \\
\hline GGBR4 & Gerdau S.A. & N1 & Siderur \& Metalur & 21.879 .186 \\
\hline GOAU4 & Metalurgica Gerdau S.A. & N1 & Siderur \& Metalur & 21.963 .123 \\
\hline MYPK4 & Iochpe-Maxion S.A. & N1 & Veiculos e peças & 674.931 \\
\hline ITAU4 & Banco Itaú Holding Financeira S.A. & N1 & Finanças e Seguros & 151.241 .430 \\
\hline ITSA4 & Itausa - Investimentos Itau S.A. & N1 & Outros & 155.145 .603 \\
\hline KLBN4 & Klabin S.A. & N1 & Papel e Celulose & 4.695 .622 \\
\hline POMO4 & Marcopolo S.A. & N2 & Veiculos e peças & 1.300 .162 \\
\hline NETC4 & Net Servicos de Comunicacao S.A. & $\mathrm{N} 2$ & Outros & 2.322 .293 \\
\hline PCAR4 & Companhia Brasileira de Distribuicao & N1 & Comércio & 10.923 .212 \\
\hline PMAM4 & Paranapanema S.A. & N1 & Siderur \& Metalur & 2.820 .585 \\
\hline RAPT4 & Randon S.A. Implementos e Participacoes & N1 & Veiculos e peças & 1.172 .553 \\
\hline SBSP3 & Cia Saneamento Basico Estado Sao Paulo & NM & Outros & 17.435.166 \\
\hline SDIA4 & Sadia S.A. & $\mathrm{N} 1$ & Alimentos e Beb & 6.522 .825 \\
\hline SCAR3 & Sao Carlos Empreendimentos e Part. S.A. & NM & Outros & 870.530 \\
\hline SUZB5 & Suzano Papel e Celulose S.A. & N1 & Papel e Celulose & 7.339 .695 \\
\hline SZPQ4 & Suzano Petroquimica S.A. & $\mathrm{N} 2$ & Química & 3.291 .157 \\
\hline TBLE3 & Tractebel Energia S.A. & NM & Energia Elétrica & 5.705 .361 \\
\hline TRPL4 & Cteep-Cia Transm Energia Eletr. Paulista & N1 & Energia Elétrica & 4.975 .229 \\
\hline UGPA4 & Ultrapar Participacoes S.A. & $\mathrm{N} 1$ & Química & 3.631 .273 \\
\hline UBBR1 1 & Unibanco Uniao Bancos Bras S.A. & N1 & Finanças e Seguros & 91.830 .733 \\
\hline UNIP6 & Unipar- Uniao de Inds. Petroquimicas S.A. & N1 & Química & 2.611 .121 \\
\hline USIM5 & Usinas Siderurgicas de Minas Gerais S.A. & N1 & Siderur \& Metalur & 18.195 .258 \\
\hline VCPA4 & Votorantim Celulose e Papel S.A. & N1 & Papel e Celulose & 9.554 .628 \\
\hline VALE5 & Cia Vale do Rio Doce & N1 & Mineração & 53.593 .732 \\
\hline WEGE4 & Weg S.A. & NM & Máquinas Indust & 3.308 .637 \\
\hline
\end{tabular}


Anomalias de calendário no mercado brasileiro: uma análise com empresas pertencentes ao IGC

\begin{tabular}{|c|c|c|c|c|c|c|}
\hline \multicolumn{7}{|c|}{ Apêndice B - Resultados para a análise do efeito dia-da-semana } \\
\hline Ação & Constante & Seg. & Ter. & Qui. & Sex. & Ret. $M$. \\
\hline ALPA4 & 0,001 & 0,001 & 0,000 & 0,000 & 0,002 & 0,426 \\
\hline ARCZ6 & 0,000 & $-0,001$ & 0,000 & $-0,001$ & $-0,001$ & 0,656 \\
\hline BBAS3 & $-0,001$ & 0,000 & 0,002 & 0,002 & 0,001 & 1,035 \\
\hline BBDC4 & $-0,001$ & 0,001 & 0,001 & 0,000 & 0,000 & 1,163 \\
\hline BRAP4 & 0,000 & 0,001 & 0,000 & 0,003 & 0,000 & 1,091 \\
\hline BRKM5 & 0,000 & $-0,001$ & 0,002 & $-0,002$ & $-0,001$ & 1,076 \\
\hline BRTO4 & 0,000 & $-0,003$ & $-0,002$ & $-0,001$ & 0,000 & 1,063 \\
\hline BRTP4 & $-0,001$ & $-0,001$ & 0,001 & 0,000 & 0,001 & 0,956 \\
\hline CCRO3 & $-0,001$ & 0,001 & 0,002 & 0,002 & 0,004 & 0,687 \\
\hline CESP5 & 0,002 & $-0,004$ & $-0,003$ & $-0,001$ & $-0,003$ & 1,142 \\
\hline CLSC6 & 0,000 & $-0,001$ & $-0,001$ & 0,001 & $-0,001$ & 0,930 \\
\hline CMIG4 & 0,000 & 0,000 & $-0,002$ & $-0,001$ & 0,000 & 1,122 \\
\hline CNFB4 & 0,000 & $-0,001$ & 0,002 & $-0,003$ & 0,002 & 0,686 \\
\hline DURA4 & 0,001 & $-0,004$ & $-0,001$ & $-0,002$ & 0,003 & 0,766 \\
\hline ELET6 & $-0,001$ & $-0,001$ & 0,000 & 0,000 & 0,000 & 1,208 \\
\hline ELPL5 & 0,000 & 0,000 & 0,001 & $-0,001$ & $-0,002$ & 1,068 \\
\hline GGBR4 & 0,000 & 0,001 & 0,000 & $-0,001$ & 0,001 & 1,146 \\
\hline GOAU4 & 0,001 & 0,000 & $-0,001$ & $-0,002$ & 0,001 & 1,083 \\
\hline ITAU4 & 0,000 & 0,000 & 0,000 & $-0,001$ & $-0,001$ & 1,174 \\
\hline ITSA4 & 0,001 & 0,000 & $-0,001$ & $-0,001$ & $-0,002$ & 1,074 \\
\hline KLBN4 & 0,001 & $-0,001$ & $-0,002$ & $-0,003$ & 0,001 & 0,933 \\
\hline MYPK4 & 0,005 & $-0,004$ & $-0,006$ & $-0,004$ & $-0,001$ & 0,493 \\
\hline NETC4 & 0,001 & $-0,005$ & $-0,002$ & 0,002 & $-0,002$ & 1,041 \\
\hline PCAR4 & $-0,001$ & $-0,001$ & 0,000 & $-0,001$ & $-0,001$ & 0,767 \\
\hline PMAM4 & 0,001 & $-0,002$ & 0,004 & $-0,002$ & 0,001 & 0,866 \\
\hline POMO4 & 0,002 & 0,000 & $-0,003$ & $-0,003$ & $-0,003$ & 0,474 \\
\hline RAPT4 & 0,002 & $-0,001$ & $-0,002$ & 0,001 & 0,000 & 0,588 \\
\hline SBSP3 & 0,002 & $-0,003$ & $-0,002$ & $-0,004$ & $-0,002$ & 0,890 \\
\hline SCAR3 & $-0,003$ & 0,006 & $-0,005$ & 0,005 & 0,014 & 0,341 \\
\hline SDIA4 & 0,001 & $-0,002$ & 0,000 & 0,000 & $-0,001$ & 0,822 \\
\hline SUZB5 & 0,002 & $-0,002$ & $-0,004$ & 0,000 & 0,000 & 0,621 \\
\hline SZPQ4 & 0,002 & $-0,003$ & 0,000 & $-0,002$ & 0,000 & 0,538 \\
\hline TBLE3 & 0,003 & 0,004 & $-0,004$ & $-0,005$ & $-0,005$ & 0,676 \\
\hline TRPL4 & 0,002 & $-0,005$ & $-0,001$ & $-0,002$ & $-0,002$ & 0,955 \\
\hline UBBR 11 & 0,001 & $-0,001$ & $-0,001$ & $-0,001$ & $-0,004$ & 1,182 \\
\hline UGPA4 & 0,001 & $-0,001$ & 0,000 & $-0,002$ & 0,000 & 0,389 \\
\hline UNIP6 & 0,000 & $-0,003$ & 0,002 & $-0,001$ & 0,000 & 0,694 \\
\hline USIM5 & 0,000 & 0,001 & 0,002 & $-0,002$ & 0,002 & 1,336 \\
\hline VALE5 & $-0,001$ & 0,002 & 0,001 & 0,001 & 0,000 & 1,021 \\
\hline VCPA4 & 0,000 & 0,000 & $-0,001$ & $-0,002$ & $-0,001$ & 0,717 \\
\hline WEGE4 & 0,002 & $-0,001$ & 0,000 & $-0,002$ & 0,001 & 0,428 \\
\hline
\end{tabular}




\begin{tabular}{|c|c|c|c|c|c|c|c|c|c|c|c|c|c|}
\hline \multicolumn{14}{|c|}{ Apêndice $\mathbf{C}$ - Resultados para a análise do efeito Janeiro } \\
\hline Ação & Const. & Fev. & Mar. & Abr. & Maio & Jun. & Jul. & Ago. & Set. & Out. & Nov. & Dez. & Ret. $M$. \\
\hline ALPA4 & 0,048 & $-0,065$ & 0,001 & $-0,034$ & $-0,002$ & 0,005 & $-0,082$ & $-0,082$ & $-0,032$ & $-0,064$ & $-0,047$ & 0,051 & 0,696 \\
\hline ARCZ6 & $-0,089$ & 0,149 & 0,092 & 0,013 & 0,128 & 0,109 & 0,115 & 0,115 & 0,065 & 0,091 & 0,103 & 0,123 & ,466 \\
\hline BBAS3 & 0 & -0 & 0 & 33 & & 0 & & 0 & 21 & 0,018 & 0,034 & -0 , & 02 \\
\hline BBDC4 & 0,013 & $-0,0$ & 0 & $-0,010$ & $-0,036$ & 3 & & & $-0,033$ & 0,025 & 0,003 & $-0,031$ & 1,172 \\
\hline BRAP4 & 0,063 & $-0,045$ & $-0,05$ & $-0,107$ & $-0,072$ & & & $-0,031$ & $-0,010$ & $-0,097$ & $-0,077$ & $-0,051$ & ,194 \\
\hline BRKM5 & $-0,084$ & 0,060 & 0,085 & 0, & & 0,089 & 1 & 0,071 & 0,061 & 0,052 & 0,080 & 0,060 & 1,239 \\
\hline BRTO4 & $-0,080$ & 0,076 & 0,049 & 0,130 & 0,023 & 0,116 & 0,033 & 0,033 & 0,066 & 0,068 & 0,136 & 0,085 & 0,636 \\
\hline BRTP4 & $-0,044$ & 0,034 & 0,030 & 0,088 & 0,026 & 0,067 & 0,005 & 0,005 & 0,028 & 0,004 & 0,073 & 0,055 & 0,456 \\
\hline $\mathrm{CCRO} 3$ & $-0,012$ & $-0,056$ & 0,038 & 0,079 & 0,037 & 0,046 & $-0,014$ & 14 & 0,045 & $-0,006$ & 0,100 & 0,015 & 0,994 \\
\hline CESP5 & $-0,023$ & 0,020 & 0,004 & 0,066 & $-0,0$ & & & & 0,105 & $-0,035$ & 0,072 & $-0,003$ & 074 \\
\hline CLSC6 & $-0,043$ & 0,015 & 0,077 & 0,052 & 0,0 & 38 & 0 , & 8 & 6 & 0 , & 18 & 0,053 & 746 \\
\hline CMIG4 & $-0,031$ & 0,016 & 0,006 & 0,124 & $-0,017$ & 0,044 & 0,031 & 0,031 & 0,021 & 0,046 & 0,022 & $-0,007$ & 1,074 \\
\hline CNFB4 & 0,020 & $-0,029$ & & 0,038 & -0 & 14 & -0 & & & & & 0,032 & 181 \\
\hline DURA4 & 0,031 & $-0,012$ & $-0,026$ & 0,007 & -0 & 4 & & -0 & 0,037 & -0 & 3 & $-0,004$ &, 862 \\
\hline ELET6 & $-0,119$ & 0,068 & 0,153 & 0,181 & 0,085 & 0,123 & 0,091 & 0,091 & 0,183 & 0,103 & 0,085 & 0,018 & 1,318 \\
\hline ELPL5 & $-0,015$ & $-0,045$ & 0,0 & 0, & -0 & & $-0,007$ & & 0,078 & -0 & 79 & 0,040 & 83 \\
\hline GGBR4 & 0,009 & 0,008 & $-0,032$ & 0,019 & $-0,020$ & 0,041 & 0,001 & 0,001 & $-0,069$ & $-0,052$ & 0,018 & $-0,002$ & 1,159 \\
\hline GOAU4 & 0,014 & $-0,006$ & 0,003 & 0,029 & $-0,026$ & 0,020 & -0 & & & $-0,032$ & 0,019 & 0,020 & 1,05 \\
\hline ITA & & & & & & & & & & & & & 52 \\
\hline ITSA4 & 0,025 & $-0,028$ & $-0,006$ & $-0,017$ & $-0,0$ & -0 & & & -0 & 0,001 & $-0,038$ & $-0,044$ & 1,094 \\
\hline KLBN4 & $-0,036$ & 0,126 & 0,091 & 0,056 & 0,081 & 0,083 & $-0,022$ & $-0,022$ & 0,074 & $-0,011$ & 0,051 & 0,001 & 0,821 \\
\hline MYPK4 & 0,022 & $-0,0$ & -0 , & -0 & 0,017 & 0 & 0 , & 0 , & 0 , & 0 , & 0,000 & 0 & , \\
\hline NETC4 & $-0,047$ & 0,011 & 0,119 & 0,117 & 0,009 & $-0,036$ & 0,026 & 0,026 & 0,115 & 0,031 & 0,105 & $-0,047$ & 0,87 \\
\hline PCAR4 & $-0,070$ & 0,026 & 0,016 & 0,015 & 0,038 & 0,072 & 0,105 & 0,105 & 0,001 & 0,099 & 0,106 & 0,103 & 0,780 \\
\hline PMAM4 & 0,000 & 0,023 & 0,036 & 0,131 & 0,010 & -0 & & -0 , & 0,042 & -0 , & & -0 & 0,882 \\
\hline POMO4 & 0,016 & $-0,027$ & $-0,043$ & $-0,069$ & $-0,008$ & 0,027 & 0,021 & 0,021 & 0,019 & $-0,042$ & -0 , & 0,099 & 0,391 \\
\hline RAPT4 & 0,013 & 0,043 & 0,024 & $-0,0$ & $-0,023$ & 0,015 & 0,035 & 0,035 & 0,039 & 0,022 & $-0,033$ & 0,022 & 0,755 \\
\hline SBSP3 & $-0,066$ & 0,064 & 0,043 & 0,122 & 0,0 & 0,097 & 0,098 & 0,098 & 0,040 & $-0,013$ & 0,092 & 0,107 & 0,797 \\
\hline SCAR3 & $-0,056$ & 0,087 & 0,009 & 0,169 & 0,016 & 0,078 & $-0,009$ & $-0,009$ & 0,085 & $-0,023$ & 0,064 & $-0,008$ & 1,125 \\
\hline SDIA4 & $-0,012$ & $-0,0$ & -0 & 0,031 & 0,026 & 0 , & 65 & 65 & 66 & 0 & 0,035 & 0,001 & 0,844 \\
\hline SUZB5 & 0,027 & $-0,0$ & -0 & $-0,055$ & $-0,017$ & $-0,025$ & 0,007 & 0,007 & 0,033 & $-0,015$ & -0 , & $-0,003$ & 0,707 \\
\hline SZPQ4 & $-0,014$ & 0,041 & $-0,020$ & 0,051 & $-0,033$ & $-0,011$ & 0,027 & 0,027 & 0,076 & $-0,004$ & 0,057 & $-0,007$ & 0,478 \\
\hline TBLE3 & 0,060 & $-0,140$ & 0,095 & 0,013 & $-0,069$ & 0,038 & $-0,1$ & -0 & $-0,064$ & $-0,028$ & $-0,125$ & $-0,006$ & 0,378 \\
\hline TRPL4 & $-0,021$ & $-0,003$ & 0,010 & 0,043 & 0,082 & 0,051 & -0, & -0 & 0,048 & 0,052 & 0,013 & 0,041 & 0,886 \\
\hline UBBR 11 & 0,012 & $-0,034$ & $-0,006$ & $-0,038$ & $-0,031$ & $-0,021$ & $-0,0$ & $-0,009$ & $-0,017$ & 0,011 & $-0,028$ & $-0,033$ & 1,282 \\
\hline UGPA4 & $-0,017$ & 0,039 & 0,062 & 0,010 & $-0,042$ & 0,073 & $-0,0$ & $-0,004$ & 0,047 & 0,015 & 0,034 & 0,017 & 0,227 \\
\hline UNIP6 & $-0,008$ & 0,014 & $-0,001$ & 0,017 & $-0,012$ & $-0,036$ & 0,022 & 0,022 & 0,001 & $-0,075$ & $-0,023$ & $-0,027$ & 0,938 \\
\hline USIM5 & 0,044 & $-0,005$ & 0,054 & $-0,097$ & $-0,084$ & $-0,020$ & 0,019 & 0,019 & $-0,048$ & $-0,064$ & 0,001 & $-0,070$ & 1,318 \\
\hline VALE5 & 0,019 & $-0,011$ & $-0,053$ & $-0,082$ & 0,004 & $-0,062$ & 0,036 & 0,036 & 0,062 & $-0,008$ & $-0,044$ & 0,004 & 0,945 \\
\hline VCPA4 & $-0,061$ & 0,132 & 0,041 & $-0,005$ & 0,079 & 0,068 & 0,116 & 0,116 & 0,084 & 0,057 & 0,072 & 0,106 & 0,334 \\
\hline WEGE & 0,037 & & -0 , & & -0 & 0,003 & & O & 52 & 0,032 & $-0,041$ & $-0,005$ & 0,322 \\
\hline
\end{tabular}


Anomalias de calendário no mercado brasileiro: uma análise com empresas pertencentes ao IGC

\section{REFERÊNCIAS}

AMIHUD, Y.; MENDELSON, H. Liquidity, asset prices, and financial policy. Financial Analysts Journal, 47, p. 56-66, nov./dec. 1991.

BACHELIER, L. Théorie de la Spéculation. Annales Scientifiques de l'Ecole Normale Superieure, I I I -17, 21-86. 1900.

BAMPI, R. E.; COLOMBO, J. A.; KLOECKNER, G. O. Análise do Desempenho de um Portfólio LongShort Formado com Base em LPA e VPA. In: XXXV ENANPAD, Anais da ENANPAD. Rio de Janeiro, 2011.

BONE, R. B.; RIBEIRO, E. P. Eficiência fraca, efeito dia-da-semana e efeito feriado no mercado acionário brasileiro: Uma análise empírica sistemática e robusta. Revista de Administração Contemporânea - RAC, v. 6, n. 1, jan./abr. 2002: 19-37.

BONOMO, M. A.; AGNOL, I. D. Retornos anormais e estratégias contrárias. In: $7^{\circ}$ Encontro Brasileiro de Finanças, Anais... São Paulo, 2007.

CAMARGOS, M. A.; BARBOSA F. V. Análise Empírica do Retorno Acionário Anormal e da Reação do Mercado de Capitais Brasileiro aos Anúncios de Fusões e Aquisições Ocorridos entre 1994 e 2001. In: XXIX ENANPAD, Anais da ENANPAD. Brasília, 2005.

CAMPOS, O. V.; LAMOUNIER, W. M.; BRESSAN, V. G. F. Lucro e os Retornos das Ações: avaliação da relevância da informação contábil. In: XXXV ENANPAD, Anais da ENANPAD. Rio de Janeiro, 2011.

CHAN, K. C. CHEN, N. Structural and return characteristics of small and large firms. Journal of Finance, 46, n. 4, p. 1467-1484, Sept. 1991.

CHAN, L. K. C.; HAMAO, Y.; LAKONISHOK, J.. Fundamentals and stock returns in Japan. Journal of Finance, 46, n. 5, p. 1739-1764, Dec. 1991.
COSTA JR., N. C. A. Sazonalidades do Ibovespa. Revista de Administração de Empresas. São Paulo, v. 30, n. 3, p. 79-84, jul./set. 1990.

COSTA JR., N. C. A.; CERETTA, P. S. Efeito dia da semana: evidência na América Latina. Teor. Evid. Econ. Passo Fundo, v. 8, n. 14 p. 27-35 maio 2000.

COSTA JR., N. C. A.; LEMGRUBER, E. F. O efeito fim de semana durante períodos de abertura e de fechamento das bolsas de valores. In: ENCONTRO ANUAL DA ANPAD, XVII, 1993, Salvador. Anais da ANPAD. Salvador: ANPAD, 1993. p. 103-110.

COSTA JR., N. C. A.; O'HANLON, J. O efeito tamanho versus o efeito mês-do-ano no mercado de capitais brasileiro: uma análise empírica. Revista Brasileira de Mercado de Capitais, Rio de Janeiro, v. 16, n. 43, p. 61-74, 1991.

ELTON, E. J., GRUBER. [et al.]. Moderna teoria de carteiras e análise de investimentos. Tradução: Antônio Zoratto Sanvicente. São Paulo: Atlas, 2004.

FAJARDO, J.; PEREIRA, R. Efeitos Sazonais no Índice Bovespa. Brazilian Business Review. v. 5, n. 3, p. 244 254, dez. 2008.

FAMA, E. F. Efficient Capital Markets: A Review of Theory and Empirical Works. The Journal of Finance, v. 25 , n. 2 , p. $383-417,1970$.

FAMA, E. F. Efficient Capital Markets: II. The Journal of Finance, v. 46. n. 5, Dec.1991.

FAMA, E. F.; FRENCH, K. R. Permanent and temporary components of stock prices. Journal of Political Economy, 96, p. 246-273, Apr. 1988.

GIBBONS, M. R.; HESS, P. J. Day of the week effects and asset returns. Journal of Business, 54, p. 579-596, 1981. 
HARRIS, L. A transaction data study of weekly and intradaily patterns in stock returns. Journal of Financial Economics, 14, p. 99-117, may 1986.

KATO, K.; SHALLEIM, J. Seasonal and size anomalies in the Japanese stock market. Journal of Financial and Quantitative Analysis, 20, n. 2, p. 243-260, June 1985.

KEIM, D. B.; STAMBAUGH, R. F.AFurther Investigation of the Weekend Effect in Stock Returns. The Journal of Finance, v. 39, n. 3, Papers and Proceedings, FortySecond Annual Meeting, American Finance Association, San Francisco, CA, December 28-30, 1983. p. 819-835, Jul. 1984.

KEIM, D. B. Trading patterns, bid-ask spreads, and estimated security returns: the case of common stocks at calendar tuning points. Journal of Financial Economics, 25, n. 1, p. 75-97, Nov. 1989.

KENDALL, M. G. The Analysis of Economic TimeSeries-Part I: Prices. Journal of the Royal Statistical Society. Series A (General), 116(1), 11-25. 1953.

LEAL, R. P.; SANDOVAL, E. B. Anomalias nos mercados de ações de países em desenvolvimento. In: ENCONTRO ANUAL DA ANPAD, XVIII, 1994, Curitiba. Anais da ANPAD. Curitiba: ANPAD, 1994. p. 213-230.

LEMGRUBER, E. F.; BECKER, J. L.; CHAVES, T. B. S. O efeito de fim-de-semana no comportamento dos retornos diários de índices de ações. In: XII Encontro Anual da ANPAD, 1988. Anais da ANPAD. Natal: ANPAD, 1998.

LIMA, J. B. N.; TERRA, P. R. S. A reação do mercado de capitais brasileiro à divulgação das informações contábeis. In: XXVIII ENANPAD, Anais da ANPAD. Curitiba, 2004.

LO, A. W. Efficient Markets Hypothesis. New Palgrave: A Dictionary of Economics, Second Edition, 2007. New York: Palgrave McMillan.
LUCENA, P.; FIGUEIREDO PINTO, A. C. Estudo de Anomalias no Mercado Brasileiro de Ações através de uma Modificação no Modelo de Fama e French. In: XXIX ENANPAD, Anais da ENANPAD. Brasília, 2005.

PAGNANI, E. M.; OLIVIERI, F. J. Instrumentos de avaliação de desempenho e risco no mercado acionário brasileiro: um estudo de anomalias de mercado na Bolsa de Valores de São Paulo (BOVESPA). Revista Brasileira de Gestão de Negócios - FECAP. Ano 6, n. 16, dez. 2004.

REINGANUM, M. Misspecifications of capital asset pricing: Empirical anomalies based on earnings yield sand market value. Journal of Financial Economics, vol. 9, p. 19-46, 1981.

SANTOS, J. O., FAMÁ, R., TROVÃO, R.; MUSSA, A. Anomalias do mercado acionário brasileiro: a verificação do efeito janeiro no IBOVESPA no período de 1969 a 2006. In: $7^{\circ}$ Encontro Brasileiro de Finanças, Anais... São Paulo, 2007.

WOOLDRIDGE, J. M. Introdução à econometria: uma abordagem moderna. Tradução: Rogério César de Souza; José Antonio Ferreira. São Paulo: Pioneira Thomson Learning, 2006. 